\section{Imaging-Spezialist präsentierte den Status Quo der dentalen Bildgebung}

Am Stand von Carestream Dental wurden den IDS-Besuchern 2015 vor allem aktuelle Entwicklungen der oralen Röntgentechnologie und neueste Prothetik-Trends (CAD/CAM) präsentiert. „Bei uns erlebten Behandler die komplette Welt der dentalen Bildgebung vom Film bis zum 3DRöntgen an“, fasst Frank Bartsch, Trade Marketing Manager bei Carestream Dental, die IDS 2015 zusammen. „Das neue CS Model aus dem CAD/CAM-System „CS Solutions“ erleichtert bei Verwendung mit dem CS 3500 Intraoralscanner das Erstellen präziser, digitaler Modelle für kieferorthopädische Anwendungen“, führt Bartsch aus. Messe-Premiere hatte auch der kompakte Intraoral-Scanner CS 7200. Mit ihm kann der Zahnarzt jetzt alle Vorteile der digitalen Speicherfolientechnologie nutzen, ohne seine gewohnten $\mathrm{Ar}$ beitsabläufe umzustellen. „Wir nennen das CS 7200 gern den ,Kleinen Bruder' des CS 7600, denn oftmals wird die umfassende Scan \& Go-Technologie des CS 7600 gar nicht richtig ausgereizt. Das CS 7200 bietet hier die gewohnte Carestreamqualität zum kleinen Preis und ergänzt unser Angebot perfekt“. Als Ausblick erwartete die Messebesucher eine besondere Ankündigung für die 2. Jahreshälfte: „Basierend auf der preisgekrönten Plattform des CS 8100 geht die Bildgebung mit dem CS

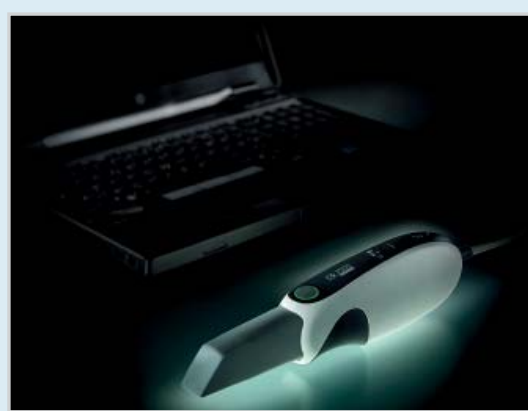

8100SC noch einen Schritt weiter. Mit diesem System erhalten Zahnärzte die Möglichkeit, ihre Praxis für umfangreichere Diagnosefunktionen um digitale Fernröntgen-Bildgebung zu erweitern - und all dies in einem kompakten System“, kündigt Bartsch an.

Nach einer Pressemitteilung der

Carestream Health Deutschland GmbH, Stuttgart

Internet: www.carestreamdental.de 